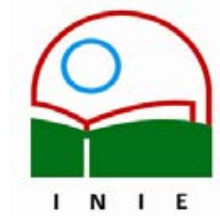

\title{
LA INVESTIGACIÓN DE LA ENSEÑANZA Y LA ENSEÑANZA DE LA INVESTIGACIÓN EN LA FORMACIÓN DOCENTE ${ }^{1}$
}

\author{
Ileana Contreras Montes de Oca, Ph.D. ${ }^{2}$
}

\section{I- INTRODUCCIÓN}

Deseo en primer término agradecer a las y los organizadores de este Congreso la oportunidad de compartir con ustedes, investigadoras e investigadores en el campo de la educación, los planteamientos que he preparado para esta conferencia, los cuales se sustentan en mis reflexiones y resultados de investigación en relación con la temática de esta actividad.

A continuación sintetizo los aspectos a los que deseo referirme en esta conferencia:

1. La urgencia de dedicar esfuerzos, recursos y condiciones apropiadas para investigar la enseñanza como condición para superar la calidad de la educación.

2. La necesidad de superar las limitaciones de las investigaciones educativas que han venido sustentando nuestro conocimiento respecto a la enseñanza.

3. La investigación colaborativa para la intervención es un enfoque apropiado para la investigación de la enseñanza y su relación con la formación docente.

4. Las condiciones que se requieren para hacer realidad e impulsar la investigación colaborativa de la enseñanza.

\footnotetext{
1 Conferencia Congreso Internacional de Investigación Educativa: "Investigar para transformar" Instituto de Investigación en Educación-Universidad de Costa Rica, COSTA RICA. 3 de febrero de 2005.

${ }^{2}$ Directora del INIE, periodo 1994-1998. icontrer@cariari.ucr.ac.cr
} 
5. Los retos y dilemas de la enseñanza de la investigación en la formación docente en el marco de los planteamientos anteriores

\section{II- PROCEDIMIENTO}

Los planteamientos a exponer se sustentan en el análisis de: los problemas, retos y expectativas en torno a la calidad de la educación formal reflejados en:

- Los documentos oficiales generados por el Ministerio de Educación Pública.

- La interpretación de los documentos oficiales relacionados con la calidad de la educación, que presentan los medios de comunicación para contribuir a formar la opinión pública en torno a la educación.

- Los planteamientos teóricos que se relacionan con las expectativas en torno a la educación escolar, tanto en el nivel nacional como en el internacional, y las perspectivas, retos y posibilidades que de ellas se derivan.

- Los resultados de investigación que hemos obtenido en torno al análisis del trabajo docente en matemática que pueden generalizarse al sistema educativo en general.

- Los enfoques con los que se ha venido estudiando la enseñanza

El criterio de presentación a utilizar es la yuxtaposición de las ideas tratadas, los resultados obtenidos y su interpretación o análisis para así apoyar los planteamientos a compartir.

III- Primer Planteamiento: La urgencia de dedicar esfuerzos, recursos y condiciones apropiadas para investigar la enseñanza como condición para superar la calidad de la educación. 
A- Conclusiones derivadas del análisis del conjunto de propuestas de cambio, relativas a las pruebas nacionales presentadas por el Ministerio de Educación Pública en el documento titulado "Relanzamiento de la Educación Costarricense ${ }^{3 "}$

Esta fuente de información resulta relevante por cuanto en la nota de presentación de este documento al Consejo Superior de Educación, el señor Ministro afirma que estos planteamientos "son producto de diferentes consultas, opiniones de profesionales internos $y$ externos del Ministerio de Educación Pública".

Presento a continuación un extracto de las consideraciones que sustentan tales propuestas:

Los resultados de las pruebas nacionales han evidenciado durante el último lustro una disminución de destrezas por parte de los educandos. Entre ellas se citan las siguientes:

- Razonamiento

- Resolución de problemas

- Aplicación del pensamiento lógico
- Interpretación

- Comprensión

- Habilidad para resumir

y redactar

Evidencian además limitaciones de los educandos en:

> El aprendizaje en general: manejo verbal, síntesis, comprensión, interpretación, análisis e inferencia textual

> En matemática: razonamiento lógico e interpretación simbólica, razonamiento analítico

El currículo de la Educación General Básica es excesivamente memorístico y enciclopédico.

La metodología pedagógica se limita a pasar la materia y no se ajusta a los cambios que requiere el proceso de globalización.

El conocimiento - que adquieren los estudiantes en la EGB- es poco significativo y duradero.

${ }^{3}$ Documento enviado por el señor Ministro de Educación Pública al Consejo Superior de Educación en oficio de fecha 29 de enero del 2004 
Se da poca atención a las variables afectivas involucradas en el aprendizaje.

En los salones de clase se promueven aprendizajes descontextualizados, pues no son congruentes con el ambiente real que viven los estudiantes.

Existe una necesidad urgente de revisar diversas variables asociadas al proceso de enseñanza y aprendizaje insertas en la propuesta curricular formal.

Se reconoce la dificultad de diagnosticar en torno a lo que ocurre en el aula debido a su complejidad, ya que en ella convergen:
$>$ El curriculum vivido
$>$ Necesidades y motivaciones
$>$ Valores, actitudes y creencias que constituyen y dan significado al aprendizaje

De tales consideraciones se puede concluir en primera instancia que:

1- En el último lustro no se han utilizado los resultados de las pruebas nacionales para tomar decisiones orientadas a superar las limitaciones que éstas reflejan - y si se ha intentado, no se ha tenido éxito- , razón por la cual podemos afirmar que las pruebas, por sí solas no han logrado cumplir con una parte muy importante de su objetivo, tal y como se enuncia en el documento mencionado:

El sistema de pruebas nacionales como un esfuerzo para medir la calidad del aprendizaje escolar con el objetivo de proveer información al sistema educativo y a la sociedad, y como un insumo para la toma de decisiones (pág 1).

2- Se reconoce además la complejidad del trabajo docente y se expone como una limitación para estudiar la enseñanza (dificultad para diagnosticar lo que ocurre en el aula). Sin embargo, se destaca la necesidad urgente de estudiar los procesos educativos que se desarrollan "revisar diversas variables asociadas al proceso de enseñanza y aprendizaje". 
3- Se evidencia la expectativa de que los procesos que se concretan en las aulas desarrollen un conjunto de destrezas tanto de aplicación general como propias de dominios específicos, como la matemática.

4- Se hace referencia a la enseñanza como una "metodología pedagógica" deficiente, que no atiende las variables afectivas, promueve aprendizajes descontextualizados, poco significativos y duraderos.

En segunda instancia, se concluye la imperiosa necesidad de estudiar sistemáticamente y con profundidad la forma en que se traduce el curriculum en las interacciones y actividades que se desarrollan en las aulas, para comprender y analizar las condiciones que explican estos resultados, de manera que las decisiones que se tomen se sustenten en el conocimiento generado.

B- Conclusiones derivadas de un estudio internacional de la enseñanza -TIMSS - para explicar el fracaso de la mayor parte de los esfuerzos que se vienen realizando en Estados Unidos para mejorar la calidad de la educación

En su libro "The teaching gap" - La brecha en la enseñanza- (99) Stigler y Hiebert afirman que a pesar de que la educación en ese país ocupa un primer lugar en sus prioridades y de que prácticamente todos los estados se encuentran desarrollando mejores estándares para orientar los aprendizajes y medios para valorar su progreso, las soluciones rápidas que hasta la fecha se han implementado no han mostrado ningún impacto importante. Ofrecen argumentos para evidenciar que el fracaso de tales esfuerzos obedece al hecho de que no ejercen impacto alguno en la enseñanza que se desarrolla en los salones de clase.

A continuación se presenta un extracto de sus planteamientos:

Sin objetivos claros no podemos tener éxito en el mejoramiento de la educación y los rendimientos académicos, pues no sabremos en qué dirección orientarnos. El establecimiento de objetivos, estándares y evaluaciones no es suficiente. 
Muchos de los esfuerzos que se realizan, a pesar de sus buenas intenciones, no tienen éxito, debido a que dejan por fuera el ingrediente que tiene mayores posibilidades de hacer una diferencia en el aprendizaje de los alumnos: La Calidad de la Enseñanza.

A pesar de que es importante tener información respecto a la calidad del aprendizaje de los alumnos, las pruebas de rendimiento, por sí mismas, no podrán nunca revelarnos cómo se pueden mejorar los rendimientos. Requerimos además de información respecto a los procesos que se desarrollan en las aulas - en la enseñanza - que contribuyen a esos rendimientos.

Desafortunadamente, quienes plantean las políticas educativas, han tomado decisiones respecto al futuro de la educación sin disponer de la información más rudimentaria acerca de qué es lo que está pasando en las aulas. Si bien se plantean marcos curriculares, si se desconoce cómo trabajan los docentes, no es posible plantear ninguna revisión curricular.

Con frecuencia, se plantean políticas educativas con la expectativa de elevar los rendimientos académicos. Si estos no se elevan - y con frecuencia este es el caso- se escuchan reclamos en el sentido de que las políticas no surtieron los efectos esperados. Nuevamente se revisan, se consultan expertos, y pronto se cuenta con nuevas recomendaciones, $y$ cambios por supuesto, generalmente en dirección opuesta.

Es relevante notar que este proceso se desarrolla sin haber recolectado un solo dato que permita determinar si el programa propuesto se llegó a implementar o no en las aulas; y si se implementó, cuán efectivo resultó para promover el mejoramiento del aprendizaje.

Si pretendemos tomar decisiones sabias, necesitamos saber qué sucede dentro de nuestras aulas.

Los políticos, reformadores, y el público en general ha subestimado seriamente el problema de cómo mejorar la enseñanza a gran escala. 
El enfoque en USA -y en Costa Rica podríamos afirmar nosotros- ha consistido en escribir y distribuir documentos de reforma $y$ pedirle a los docentes que implementen las recomendaciones que se plantean en tales documentos. Quienes han trabajado en este problema saben bien que ese enfoque no funciona.

La profesión de enseñanza no cuenta con información suficiente respecto a qué constituye "enseñanza efectiva", y los docentes no cuentan con los medios para compartir con éxito ese conocimiento.

Para realmente mejorar la enseñanza, debemos invertir mucho más que lo que hemos hecho, generando y compartiendo conocimiento acerca de la enseñanza.

C- Perspectivas de los responsables de la educación matemática respecto a la problemática existente en ese campo y los factores que inciden en ellos.

Como parte de un estudio $^{4}$ que realicé alrededor del trabajo docente de los profesores de matemática en la educación secundaria se identificaron las perspectivas de los formadores de docentes, asesores ministeriales y profesores en servicio alrededor de los problemas de la educación matemática en el país y sus determinantes.

A continuación se presentan algunos de los resultados obtenidos, los cuales ilustran no sólo la necesidad de profundizar en el conocimiento de la enseñanza sino la tendencia predominante a hacer recaer en los docentes la responsabilidad por las deficiencias educativas y el bajo rendimiento obtenido en las "evaluaciones de la calidad educativa":

Los formadores de docentes consultados ubicaron entre los principales factores que inciden en la problemática a:

${ }^{4}$ Contreras, I. (2002) "La educación matemática en la escuela secundaria: mecanismos de resistencia a un modelo agotado" Proyecto No. 724-97-347 
> Los profesores que laboran en la educación secundaria: porque "de ellos depende la enseñanza"; su actitud hacia ella genera actitudes negativas en los estudiantes hacia los contenidos curriculares; existen limitaciones en su formación, actualización y capacitación profesional; sus condiciones laborales aunadas a sus problemas de formación en la didáctica de la matemática

Los profesores no saben qué es hacer una educación matemática. Para su trabajo dependen del libro del texto, al cual siguen fielmente

- Los problemas metodológicos del enfoque utilizado en su enseñanza:

Los asesores ministeriales señalaron el bajo rendimiento reflejado en las pruebas como uno de los problemas y entre los factores que lo explican anotan:

> La cultura del aula que se caracteriza por un trabajo basado en recetas, que si bien permite resolver dificultades inmediatas no estimula el razonamiento y la capacidad de resolución de problema.

$>$ Un enfoque metodológico -enseñanza- que promueve aprendizajes a corto plazo, y que se orienta a la preparación para el examen. No hay articulación de los contenidos entre sí ni con sus ámbitos de aplicación. Esto genera desmotivación y apatía en los estudiantes.

$>$ Las condiciones en que laboran los docentes, y concretamente los nombramientos por lección, tornan invisible una parte importante de su trabajo docente.

> Las deficiencias detectadas en la formación docente en relación con el planeamiento, el manejo de los temas, y su didáctica. 
Los profesores en ejercicio consultados mencionaron, entre los determinantes de la problemática, a los siguientes:

$>$ El sistema de evaluación de los aprendizajes vigente encasilla al docente

> La expectativa Ministerial para que la enseñanza responda a un enfoque constructivista pero sin ofrecer a los docentes las condicione necesarias para su aplicación - materiales y esfuerzos necesarios -. El enfoque esperado no responde a la realidad diaria de las aulas.

- La desmotivación de los docentes producto de la responsabilidad que se les atribuye por el fracaso de sus estudiantes y la culpabilidad que les genera verificar que fallaron sus esfuerzos por "transmitir" los contenidos programáticos. Al respecto afirman:

En los periódicos, la prensa generalmente informa sobre los resultados nada más, pero no informa sobre lo que realmente uno hace en el aula, ni los esfuerzos que el docente hace para tratar de mantener a 40 ó 45 estudiantes dentro de un aula. Nada más dicen de tal colegio aprobaron el examen tantos, listo, no dicen ni cuantos alumnos tenía uno, ni en qué condiciones trabajo, ni si era o no factible obtener otros resultados.

$Y$ en efecto, en la prensa es frecuente encontrar, artículos como el siguiente: 
La Nación, Jueves 7 de marzo de 2002

Exámenes de bachillerato y $9^{\circ}$ en el 2001

Pruebas revelan fallas educativas

- Sindicatos y pedagogos contra esos exámenes

"Félix Barrantes, director de Control de Calidad del MEP, explicó que estos informes son una buena guía para que los colegios corrijan sus debilidades.

Empero, Rafael Jiménez insistió en que se deben aplicar otros instrumentos, como mejor capacitación a los profesores."

\section{D- $\underline{\text { Perspectivas teóricas }}$}

El conocimiento teórico acerca del desarrollo del niño ha continuado creciendo, sin embargo, la forma de relacionar este conocimiento con los contextos prácticos en los cuales los adultos intencional y sistemáticamente intervienen para propiciar este desarrollo, o para decirlo con una palabra, para educar, continúa casi tan misterioso como cuando se iniciaron tales esfuerzos (Olson, D.R. y Bruner, J.S, 1996, p. 9)

La manera tradicional de plantear las relaciones entre el "conocimiento teórico" y los contextos prácticos de educación" ha consistido en adoptar una teoría, un enfoque o un planteamiento global de la educación, de la enseñanza, del desarrollo, del aprendizaje, o incluso del funcionamiento psicológico, como punto de partida para formular orientaciones y prescripciones sobre cómo deben planificarse y llevarse a cabo los procesos de enseñanza y aprendizaje en las aulas, asumiendo que de su correcta interpretación y aplicación se obtendría una mejora substancial de dichos procesos (Coll, C., 1999). 
Sin embargo, la complejidad de las prácticas escolares evidencia la limitación de abordar su estudio desde una sola perspectiva teórica. Tales prácticas se caracterizan por la multidimensionalidad suceden muchas cosas-; la simultaneidad - al mismo tiempo-; la inmediatez - con mucha rapidez-; la impredictibilidad - suceden cosas inesperadas y no planificadas-; la publicidad - las acciones de los participantes son públicas; la historia - lo que sucede hoy responde en parte a lo que ha sucedido anteriormente (Doyle, 1986).

De ahí que derivar principios metodológicos y propuestas de actuación de una teoría generada al margen del contexto institucional escolar, con el propósito de contribuir a su mejoramiento, como se ha hecho con las teorías psicológicas, es una simplificación de un proceso complejo que no sólo genera distorsiones de la teoría, sino que además estos psicologismos han culminado en el desprecio de la tarea educativa del docente y de la misma pedagogía (Bixio, C., 2002; Coll, C. 1999; Eisenhart, M. y Borko, H., 1993).

Las instituciones de educación formal constituyen el contexto educativo en el que la cultura media el desarrollo socio-cognitivo (Cole, 1985). Se trata de un contexto tanto físico como socio-cultural que integra un conjunto de normas, valores, relaciones, actitudes y conductas, que conciernen a una pluralidad de grupos sociales, cuyos vínculos recíprocos regulan. Este constituye el campo social en que sus agentes le dan sentido a sus prácticas, incidiendo en los procesos de enseñanza aprendizaje y los conocimientos que se generan.

De ahí que, toda "intervención" que se pretenda realizar en las organizaciones escolares deba apoyarse en el análisis de las relaciones y prácticas implicadas en los procesos de enseñanza, en el entendido que estos no se reducen a las relaciones directas educador - educando, ya que esta pareja es sólo el elemento terminal de toda estructura escolar, cuyo encadenamiento colectivo es cada vez más largo y complejo (García, 1995; Bourdieu, 1991, Lourau, 1994). 
Todo intento de superar el nivel de propuesta conceptual, y en consecuencia, la dicotomía tradicional entre lo teórico y lo práctico, con aportes cuyo nivel de abstracción trascienda la "visión social" (Gore, 1996) de manera que se articulen estrategias docentes, prácticas, modelos, o componentes programáticos viables, deberá reconocer con acciones propias del análisis institucional, que la práctica educativa, en tanto institucionalizada, no empieza desde cero, sino que está histórica y localmente situada, y constituye un proceso que por lo general y, para cada docente o para cada proyecto e idea nueva, está ahí funcionando. Quien pretenda modificar esa práctica, deberá subirse "en marcha" al proceso. La innovación se traduce entonces en un intento de "corrección de trayectoria" para abrir nuevos sistemas de posibilidad en las discontinuidades de las pautas que organizan las relaciones y prácticas institucionales (Gimeno, 1997).

En consecuencia, por excelente que sea la calidad de las teorías acerca del aprendizaje, no nos eximen de la necesidad de realizar investigaciones para construir y generar las estrategias didácticopedagógicas que dichas teorías no pueden proporcionarnos.

A pesar de ello, hemos presenciado la traslación de las teorías del aprendizaje al aula, sin investigaciones que atiendan cómo se modifica el proceso enseñanza-aprendizaje (que implica dos procesos distintos pero interdependientes) en situación, en una institución con pautas que regulan a su vez los procesos de enseñanza aprendizaje. Como resultado de ello, nos encontramos frente a nuevas expectativas para la enseñanza que se realiza en las aulas, las cuales responden a nuestra mayor comprensión de los aprendizajes. Esta comprensión nos permite a lo sumo caracterizar los aprendizajes esperados, y ofrecer indicaciones generales para facilitarlos, y sin embargo, insistimos en evaluar y mejorar la enseñanza al margen de una comprensión de la enseñanza y de la construcción de las estrategias para desarrollarla.

IV- Segundo Planteamiento: La necesidad de superar las limitaciones de las investigaciones educativas que han venido sustentando nuestro conocimiento respecto a la enseñanza 
El estudio del proceso educativo - enseñanza y aprendizaje- , en los contextos escolares implica una observación que $\mathrm{NO}$ es pasiva, sino que constituye una lectura desde una perspectiva - referente conceptual- o forma de entender las relaciones enseñanzaaprendizaje que la organiza y permite interpretar lo observado. De ahí que la selección de las unidades a analizar, los instrumentos para recoger la información, y las categorías utilizadas para su análisis deben ser coherentes con la perspectiva de observación en que se sustenta el estudio.

Sin embargo, gran parte de los resultados educativos ya comentados se apoyan en evaluaciones que responden a perspectivas centradas en el aprendizaje esperado, que renuncian a comprender cómo orientar su enseñanza en los contextos específicos evaluados, dejando un vacío en torno a los resultados necesarios para mejorarlos.

Para comprender la enseñanza es necesario atender las preguntas y dilemas que enfrentan los participantes del proceso educativo y la complejidad de las prácticas escolares. Los distintos programas de investigación dan cuenta de las perspectivas privilegiadas.

El estudio de la enseñanza desde las perspectivas teóricas propuestas para su estudio se plantea como el análisis de las interacciones educativas que representan una acción simultánea y recíproca de los participantes en contextos institucionales determinados, en torno a contenidos de aprendizaje que implican a su vez determinadas tareas, con el objetivo de lograr determinados fines. Incluye componentes comunicacionales, intencionales y contextuales (físicos, tecnológicos y contextuales.

V- Tercer Planteamiento: La investigación colaborativa para la intervención es un enfoque apropiado para la investigación de la enseñanza y su relación con la formación docente

Este tipo de enfoque investigativo pretende superar la brecha existente entre investigadores y docentes, mediante la participación activa de las docentes, por medio del diálogo y la negociación, en la identificación y análisis de los problemas (Eisenhart y Borko, 1993; 
Florio-Ruane, 1989). Se orienta además a promover en forma deliberada y sistemática el cambio de las prácticas existentes.

Pretende superar los aspectos que se han identificado como verdaderos obstáculos para el diseño y desarrollo de investigaciones en el contexto escolar orientadas a generar resultados que puedan incorporarse tanto en las prácticas de trabajo en el aula, como en las políticas educativas.

Dichos obstáculos se describen a continuación:

1. Las diferencias reales, propias de la organización del trabajo de investigadores y docentes, que explican las distintas perspectivas, preocupaciones, creencias, y valores, entre uno y otro grupo.

Dichas diferencias se manifiestan en el carácter que tienen en cada grupo, las responsabilidades propias de su respectivo trabajo profesional, los incentivos con que cuentan, el tiempo de que disponen para el análisis de los problemas a enfrentar, y las formas utilizadas para determinar y justificar lo que resulta más importante de atender.

Por lo tanto, estas condiciones propician distintas maneras de identificar los problemas de la clase, y de proponer e implementar las soluciones.

2. Los investigadores generalmente tienden a simplificar la complejidad del trabajo docente en el aula y las presiones que éste genera.

Las aulas poseen características propias que inciden en sus participantes y que demandan la toma de decisiones en sitio por parte del docente, independientemente de su perspectiva educativa, y de sus formas de organización de los estudiantes para el aprendizaje. Doyle (1986).

Aquellas investigaciones que simplifican o ignoran estas características de las aulas, difícilmente pueden generar resultados que respondan a las preocupaciones e intereses de 
los maestros, y por lo tanto, cuentan con pocas posibilidades de ser incorporadas por el docente en las actividades que desarrolla en el aula.

3. Las diferentes perspectivas respecto al valor de los resultados que tienen investigadores y docentes.

Para los investigadores la relevancia de la investigación generalmente depende de: sus implicaciones para la comprensión de repercusiones de amplio alcance; para el mejoramiento y predicción de resultados futuros; para sustentar políticas educativas; para fortalecer los programas de investigación en los que se inscriben, y publicar sus resultados, entre otros.

Los docentes por otra parte, esperan de la investigación resultados que les permitan apoyar directamente su práctica de clase y superar los problemas que enfrentan en su trabajo docente cotidiano.

VI- Cuarto Planteamiento: Las condiciones que se requieren para hacer realidad e impulsar la investigación colaborativa de la enseñanza

De lo expuesto se desprende que hacer realidad la investigación colaborativa de la enseñanza demanda:

1. Condiciones laborales que permitan la integración de equipos que además de interdisciplinarios integren a los participantes directos en los procesos estudiados.

2. Un diseño que contemple fases de negociación de las formas de participación y de preparación de los integrantes para construir un referente común.

3. Difusión y seguimiento de los trabajos realizados.

4. Evaluación de cada proyecto para integrar un programa vigoroso que progresivamente se fortalezca.

5. Integrarse en un verdadero sistema educativo. 
VII- Quinto Planteamiento: Los retos y dilemas de la enseñanza de la investigación en la formación docente en el marco de los planteamientos anteriores

1. La formación docente debe sustentarse en la investigación de los procesos educativos y reflejar las expectativas planteadas para aquellos. Para ello tiene el reto de integrar equipos de investigación de la enseñanza que respondan al enfoque colaborativo para abordar en primera instancia el propio proceso de formación docente, y posteriormente el estudio de los procesos de investigación de la enseñanza.

2. La enseñanza de la investigación en la formación docente es una condición necesaria pero no suficiente para el mejoramiento de los procesos educativos. Requiere que se inscriba en contextos estrechamente relacionados con los ámbitos laborales en que se desempeñarán los docentes.

Necesita además que se apoye en perspectivas teóricas derivadas de diversas investigaciones, es decir, que se integre en verdaderas líneas y programas de investigación de la enseñanza de tal manera que permita construir una historia o "saber progresivo". Sólo así las intervenciones o innovaciones que se sustenten en ella, estarán respaldadas por la producción teórica generada en el campo.

3. Por lo tanto, la enseñanza de la investigación en la formación docente debe repensarse de manera que supere la perspectiva de caja de herramientas que la ha caracterizado. Esto no significa que no deba pretender que los docentes se apropien de herramientas y técnicas de investigación, sino que estas deben adquirir significado y relevancia en el marco de los contextos en que se realiza y desde las perspectivas teóricas que las sustentan.

4. La enseñanza de la investigación en el marco de una formación docente orientada a depositar en los futuros docentes la tarea de comprender la enseñanza para responder a los planteamientos generales que se demandan para lograr los aprendizajes prescritos; para un ejercicio laboral en donde no se 
ofrecen condiciones laborales para desarrollarla, ni espacios reales de participación o "colaboración" en las investigaciones realizadas desde los centros de investigación; y en donde esta debe reducirse a uno o dos cursos de formación, deberá limitarse a preparar para la presentación de un trabajo final de graduación, o para acceder a estudios de posgrado; y a lo sumo a identificar, valorar y derivar principios de las investigaciones realizadas por otros.

\section{REFERENCIAS}

Bixio, C. (2002). Enseñar a aprender: Construir un espacio colectivo de enseñanza-aprendizaje. Argentina: Homo Sapiens Ediciones.

Bourdieu, P. (1991). El sentido práctico. Madrid: Taurus.

Cole, M. (1985). The zone of proximal development: where culture and cognition create each other. En_J.V. Wertsch (comp.). Culture, Communication and Cognition: Vygostskian Perspectives. Cambridge: Cambridge University Press.

Coll, C. (1999). La concepción constructivista como instrumento para el análisis de las prácticas educativas. En Psicología de la instrucción y el aprendizaje en la educación secundaria. Barcelona: Horsori

Doyle, W. (1986). Classroom organization and management. En M.C. Wittrock (ed.) Handbook of Research on Teaching ( $3^{\text {a }}$. ed.) pp.392-431. New York: Macmillan.

Eisenhart, M. y Borko, H. (1993). Designing classroom research. Boston: Allyn \& Bacon.

Florio-Ruane, S. (1989). Research, recalibration and conversation: A response to Cazden, Diamondstone, and Naso. En The Quaterly of the National Writing Project and the Centre for the Study of Writing. V.11 n.4: 4-6, 24. 
García, F.J. (1995). Análisis del sentido de la acción: El trasfondo de la Proyecto de Gobernabilidad Democrática para Centroamérica intencionalidad. En Juan Manuel Delgado y Juan Gutiérrez (Coord.) Métodos y técnicas cualitativas de investigación en ciencias sociales, de. Madrid: Síntesis.

Gimeno, J. (1997). Docencia y cultura escolar: Reformas y modelo educativo. Buenos Aires: Lugar Editorial.

Gore, J.M. (1996). Controversias entre las pedagogías. Madrid: Motara.

Lourau, R. (1994). El análisis institucional. Buenos Aires: Amorrortu editores

Olson, D.R. y Bruner, J.S. (1996). "Folk psychology and folk pedagogy. En D. R. Olson y N. Torrance (Ed.). The Handbook of Education and Human Development Cambridge, MA: Blackwell, p.9

Stigler, J. y Hiebert, J. (1999). The teaching gap. New York: The Free Press. 